\title{
High Temperature Behavior of Subpicosecond Electron Transport Transient in 3C- and 6H-SiC
}

\author{
E. F. Bezerra, E. W. S. Caetano, V. N. Freire, \\ Departamento de Física, Universidade Federal do Ceará, \\ Caixa Postal 6030, Campus do Pici, 60455-760 Fortaleza, Ceará, Brazil \\ E. F. da Silva Jr., \\ Departamento de Física, Universidade Federal de Pernambuco, \\ Cidade Universitária, 50670-901 Recife, Pernambuco, Brazil \\ and J. A. P. da Costa \\ Departamento de Física Téorica e Experimental, Universidade Federal do Rio Grande do Norte, \\ Caixa Postal 1641, 59072-970 Natal, Rio Grande do Norte, Brazil \\ Received February 8, 1999
}

\begin{abstract}
A study of the subpicosecond high-field electron transport transient in $3 \mathrm{C}$ - and $6 \mathrm{H}-\mathrm{SiC}$ polytypes at high lattice temperatures is performed. Electric field intensities up to $1000 \mathrm{kV} / \mathrm{cm}$ and lattice temperatures of 300,673 , and $1073 \mathrm{~K}$ are considered. It is shown that the transient regime behavior depends on the electric field intensity as well as on the lattice temperature, but it is always shorter or of the order of $0.3 \mathrm{ps}$. For the lowest lattice temperature, an electron velocity overshoot can always occur when the electric field intensity is higher than $300 \mathrm{kV} / \mathrm{cm}$, but it decreases and can even disappear when the lattice temperature is raised.
\end{abstract}

\section{Introduction}

In the past decade, we have witnessed the growing technological importance of silicon carbide ( $\mathrm{SiC}$ ) as a new material with a number of physical and electrical properties which surpasses, in certain conditions, those of crystalline silicon. The use of $\mathrm{SiC}$ in new generations of advanced devices is stimulated by its high stability, strong chemical bonding, large thermal conductivity and breakdown fields, high saturation velocity and low leakage current. SiC may be grown in either cubic or hexagonal crystalline forms, presenting a large variety of polytypes $(3 \mathrm{C}-, 2 \mathrm{H}-, 4 \mathrm{H}-$ and $6 \mathrm{H}-\mathrm{SiC}$ are some of the most common forms). Among the wide range of applications of $\mathrm{SiC}$ polytypes are suitable for high-power, high-temperature, high-frequency, radiation-hard, and light-emitting devices. Ab initio studies of $\mathrm{SiC}$ electronic properties showed that their polytypes present a wide range of band gaps (1.27-3.36 eV) and different values for the carrier effective mass as a result of significant differences in their band structures [1]. 3C-SiC $\left(E_{g}=2.39 \mathrm{eV}\right)$ and $6 \mathrm{H}-\mathrm{SiC}\left(E_{g}=2.86 \mathrm{eV}\right)$ have been more extensively studied due to potential applications associated to their higher saturation velocities, particularly in the domain of high-temperature and highspeed/high-field nanostructures and devices circuitry. On the other hand, non-stationary physical conditions are often imposed on the electron transport mechanisms, which lead to electron drift velocity and energy time transients before steady-state transport conditions are attained.

Cubic SiC high-field transport in the steady-state was studied more than two decades ago by Ferry [2] through the balance equation method. In recent years, a number of new and interesting SiC high-field electron transport studies have been addressed by several authors [3-8]. Steady-state transport behavior at room temperature was studied in 3C-SiC using Monte Carlo simulation within a single equivalent isotropic valley picture [3], and considering both parabolic and nonparabolic band schemes. It was shown that the electron drift velocity presents a gentle peak at electric fields around $400 \mathrm{kV} / \mathrm{cm}$ in the nonparabolic band case. Similarly, Monte Carlo calculations [4, 5] of electron 
transport in $4 \mathrm{H}-\mathrm{SiC}$ indicate a mobility reduction as the temperature is increased in the $100-700 \mathrm{~K}$ range. Other studies performed using the Monte Carlo method [6, 7] further investigated the steady-state transport properties in $6 \mathrm{H}_{-}, 4 \mathrm{H}^{-}$, and $3 \mathrm{C}-\mathrm{SiC}$, mainly at room temperature. The hydrodynamic balance equation method was recently used to study the high temperature hot electron steady-state transport behavior in $3 \mathrm{C}$ - and $6 \mathrm{H}-\mathrm{SiC}$ [8]. The calculations were performed within the single equivalent isotropic valley approach for lattice temperatures of 300,600 , and $1073 \mathrm{~K}$, and have also considered band nonparabolicity. The behavior of the electron drift velocity and energy with the external electric field indicate a shift at high fields of a smooth peak in the drift velocity-electric field relation as the temperature is raised. In all of these works, the main scattering mechanisms considered in determining the steadystate high-field electron transport in the $4 \mathrm{H}-, 6 \mathrm{H}-$, and $3 \mathrm{C}-\mathrm{SiC}$ polytypes were the acoustic deformation potential, polar-optical phonon, intervalley phonon, ionizedimpurity and impact ionization.

To date, most of the investigations on the SiC highfield transport were restricted to steady-state phenomena, principally the determination of the saturated electron drift velocity. However, it is also of scientific and technological interest to have information on the ultrafast transport transient properties of silicon carbide polytypes. This need arises because $\mathrm{SiC}$ thermal stability at high temperatures is a remarkable characteristic for advanced technological applications, while the subpicosecond time response to high electric fields make SiC presently to be one of the most important semiconductors for high-frequency applications in high power and high temperature operating devices.

In this work, it is presented a study of high lattice temperature effects on the subpicosecond transient behavior of the electron drift velocity and energy in $3 \mathrm{C}$ - and $6 \mathrm{H}-\mathrm{SiC}$ as they attain their steady-state. Boltzmann-like transport equations in the momentum and energy relaxation time approximation are used to calculate the time evolution of the mean electron drift velocity and energy within a single equivalent isotropic valley picture for both polytypes. Acoustic, polar optical and intervalley phonons, as well as neutral, ionized impurities and impact ionization are the main electron scattering mechanisms considered in the transport calculations. The transient regime is shown to be shorter than $0.3 \mathrm{ps}$ for electric fields up to $1000 \mathrm{kV} / \mathrm{cm}$. For the lowest lattice temperature, an overshoot in the electron drift velocity always occurs when the applied electric field intensity is higher than $300 \mathrm{kV} / \mathrm{cm}$.

\section{Model and transport equa- tions}

The model used for the $3 \mathrm{C}$ - and $6 \mathrm{H}-\mathrm{SiC}$ polytypes during the calculations performed in this study consider a simple isotropic valley picture for their band structures [3]. The electron effective mass in the equivalent conduction band $\mathrm{m}_{c}$ is taken as $0.346 \mathrm{~m}_{0}$ for $3 \mathrm{C}-\mathrm{SiC}$ and $0.3 \mathrm{~m}_{0}$ for $6 \mathrm{H}-\mathrm{SiC}$, where $\mathrm{m}_{0}$ is the free electron mass. This procedure has been successfully adopted in a recent Monte Carlo simulation of the $3 \mathrm{C}-\mathrm{SiC}$ highfield steady-state transport at $300 \mathrm{~K}$, and in the high temperature hot electron steady-state transport calculations for $6 \mathrm{H}$ - and $3 \mathrm{C}$-SiC which have being performed using the hydrodynamic balance equation method [3, 8]. In addition, the model accounts for band nonparabolicity through the hyperbolic approximation, in which the following relation is valid: $[3,8,9]$

$$
\frac{\hbar^{2} k^{2}}{2 m_{c}}=\varepsilon(1+\alpha \varepsilon),
$$

where $\alpha$ is the nonparabolic band coefficient, which is $0.323 \mathrm{eV}^{-1}$ for $3 \mathrm{C}-\mathrm{SiC}$, and $\alpha=\left(1-m_{c} / m_{0}\right)^{2}$ for $6 \mathrm{H}-\mathrm{SiC}[8]$.

The time evolution of the mean electron drift velocity $v(t)$ and energy $\varepsilon(t)$ is obtained by solving numerically their two coupled Boltzmann-like transport equations in the momentum and energy relaxation time approximation, as given below:

$$
\begin{gathered}
\frac{d v(t)}{d t}=\frac{q E_{F}}{m_{c}}-\frac{v(t)}{\tau_{p}(\varepsilon)}, \\
\frac{d \varepsilon(t)}{d t}=q v(t) E_{F}-\frac{\varepsilon(t)-\varepsilon_{L}}{\tau_{\varepsilon}(\varepsilon)},
\end{gathered}
$$

where $E_{F}$ is the electric field intensity; $\varepsilon_{L}=3 k_{B} T_{L} / 2$ is the average electron thermal energy at the lattice temperature $T_{L} ; \tau_{p}(\varepsilon)$ and $\tau_{\varepsilon}(\varepsilon)$ is the momentum and energy relaxation time, respectively; $k_{B}$ is the Boltzmann constant, and $q$ is the electric charge of the electron.

The calculations are performed for electric fields of 200,600 , and $1000 \mathrm{kV} / \mathrm{cm}$. They are assumed to be applied in the $<111>$ direction for the sake of disregarding electron redistribution among equivalent valleys, and to minimize the electron population in higherlying subsidiary minima. The relaxation times $\tau_{p}(\varepsilon)$ 
and $\tau_{\varepsilon}(\varepsilon)$ are obtained through the steady-state relations $v \times E_{F}$ and $\varepsilon \times E_{F}$ determined for $3 \mathrm{C}-\mathrm{SiC}$ and $6 \mathrm{H}-\mathrm{SiC}$ at the lattice temperatures $T_{L}=300 \mathrm{~K}$, $673 \mathrm{~K}$, and $1073 \mathrm{~K}$, as described elsewhere [8]. Therefore, the electron scattering mechanisms considered here are acoustic-deformation-potential, polar-optical phonon, intervalley phonon, and ionized impurity scattering.

The use of the steady-state relations $v \times E_{F}$ and $\varepsilon \times E_{F}$ to calculate the relaxation times $\tau_{p}(\varepsilon)$ and $\tau_{\varepsilon}(\varepsilon)$ is a successful scheme pioneered by Shur [10], which was thereafter used by other researchers [11, 12], either to study transient behavior of carriers in GaAs or as a way to save computational time during the modeling of a submicrometer gate field-effect transistor including effects of nonsteady-state dynamics. Recently, this technique allowed the study of high-magnetic-field effects on the terahertz mobility of hot electrons in $n$-type InSb [13]. While Monte Carlo transport simulations of the transient regime is very time consuming, the approach used here to solve Eqs. (2) and (3) requires very short computation time since at most a standard Runge-Kutta scheme of fourth order is necessary for their numerical solution.

\section{Results and Discussions}

Fig. 1 and 2 (Fig. 3 and 4) present, respectively, the time evolution of the mean drift velocity and energy of electrons in $3 \mathrm{C}-\mathrm{SiC}(6 \mathrm{H}-\mathrm{SiC})$ at lattice temperatures of $300 \mathrm{~K}$ (top), $673 \mathrm{~K}$ (middle), and $1073 \mathrm{~K}$ (bottom). The curves were obtained for electric field intensities of $200 \mathrm{kV} / \mathrm{cm}$ (dotted lines), $600 \mathrm{kV} / \mathrm{cm}$ (dashed lines), and $1000 \mathrm{kV} / \mathrm{cm}$ (solid lines). The transient behavior of the mean drift velocity and energy is always shorter than $0.3 \mathrm{ps}$ for both $\mathrm{SiC}$ polytypes, being a little bigger in $3 \mathrm{C}-\mathrm{SiC}(\sim 0.3 \mathrm{ps})$ than in $6 \mathrm{H}-\mathrm{SiC}(\sim 0.2 \mathrm{ps})$. With the lattice temperature increasing from $300 \mathrm{~K}$ to $1073 \mathrm{~K}$, one can observe a strong reduction of the maximum values for both the electron mean drift velocity and energy, which is a consequence of the scattering mechanisms strength.

For high enough electric fields, the mean electron drift velocity in both polytypes presents an overshoot, as shown in Figs. 1 and 3. At $300 \mathrm{~K}$, the electron velocity overshoot appears when the electric field intensity is higher than $300 \mathrm{kV} / \mathrm{cm}$. However, the overshoot effect is substantially reduced when the lattice temperature increases, and can even disappear when the lattice

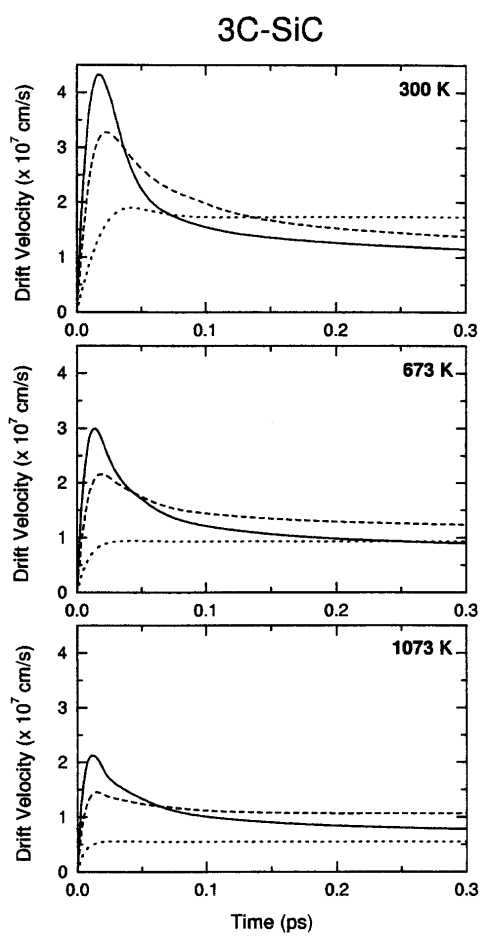

Figure 1. Subpicosecond behavior of the mean electron drift velocity in 3C-SiC for lattice temperatures of $300 \mathrm{~K}$ (top), $673 \mathrm{~K}$ (middle), and $1073 \mathrm{~K}$ (bottom). The applied electric field intensities are $200 \mathrm{kV} / \mathrm{cm}$ (dotted line), $600 \mathrm{kV} / \mathrm{cm}$ (dashed line), and $1000 \mathrm{kV} / \mathrm{cm}$ (solid line).

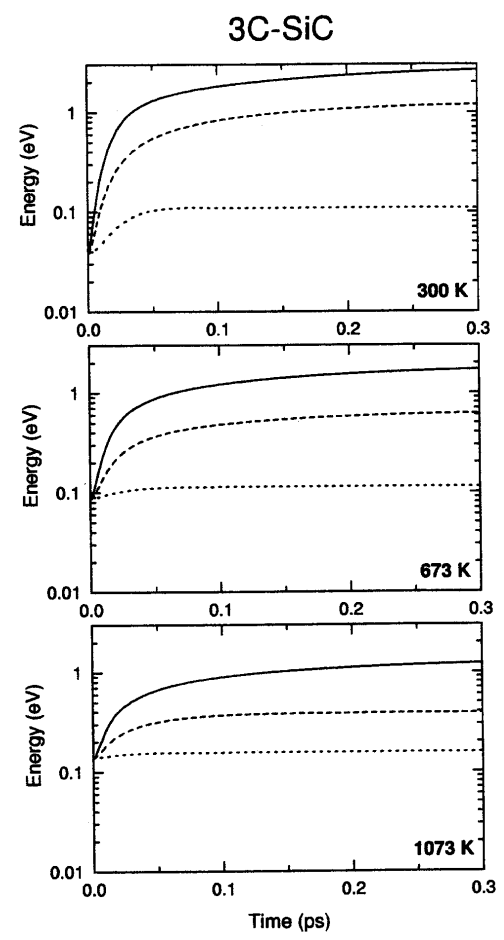

Figure 2. Subpicosecond behavior of the mean electron energy in $3 \mathrm{C}$-SiC for lattice temperatures of $300 \mathrm{~K}$ (top), $673 \mathrm{~K}$ (middle), and $1073 \mathrm{~K}$ (bottom). The applied electric field intensities are $200 \mathrm{kV} / \mathrm{cm}$ (dotted line), $600 \mathrm{kV} / \mathrm{cm}$ (dashed line), and $1000 \mathrm{kV} / \mathrm{cm}$ (solid line). 
temperature is high enough (see dotted lines in both the figures). For a given electric field and instant of time, $v(t)$ is always higher in $3 \mathrm{C}-\mathrm{SiC}$ than in $6 \mathrm{H}-\mathrm{SiC}$. This is particularly true in the velocity overshoot case, which is more pronounced in the former than in the latter. It has been suggested that an overshoot effect in the electron drift velocity may occur for low fields only when the mean momentum relaxation rate of the electron is larger than its mean energy relaxation rate $[14,15]$. The overshoot effect can also be due to the intervalley transfer mechanism and the related change in the electron effective mass associated to the intervalley scattering process $[14,15]$.

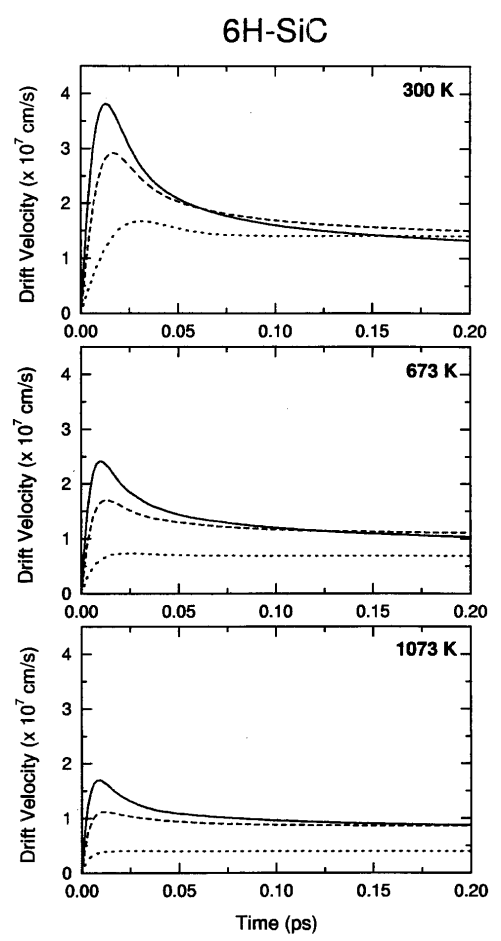

Figure 3. Subpicosecond behavior of the mean electron drift velocity in $6 \mathrm{H}-\mathrm{SiC}$ for lattice temperatures of $300 \mathrm{~K}$ (top), $673 \mathrm{~K}$ (middle), and $1073 \mathrm{~K}$ (bottom). The applied electric field intensities are $200 \mathrm{kV} / \mathrm{cm}$ (dotted line), $600 \mathrm{kV} / \mathrm{cm}$ (dashed line), and $1000 \mathrm{kV} / \mathrm{cm}$ (solid line).

Fig. 2 and 4 depicts, respectively, the time evolution of the mean electron energy in $3 \mathrm{C}-\mathrm{SiC}$ and $6 \mathrm{H}-\mathrm{SiC}$ towards the steady state. When the electric field intensity is low, the energy gained by the electrons from the heating action of the electric field (see the first term in the right side of Eq. (3)) is mainly transferred to the phonons through the energy dissipation term (second term in the right side of Eq. (3)). Since at the beginning the momentum dissipation is small, the growth rate of the mean electron drift velocity is higher than the growth rate of the mean electron energy, and consequently $v(t)$ increases faster than $\varepsilon(t)$. For high field intensities, the dissipative term is not strong enough to drain the excess energy transmitted from the electric field to the electrons. As a consequence, this excess is retained as electron thermal energy, and the growth rate of the electron mean energy increases strongly. At high lattice temperatures, the variation of the growth rate of the mean electron energy is smaller because the electron energy dissipation is stronger.

Finally, it is worth to highlight the long term behavior of both the mean electron drift velocity and energy presented in Figs. 1-4 is in very good agreement with steady-state calculations performed either through Monte Carlo simulations or using the hydrodynamic balance equation method $[8,6]$.

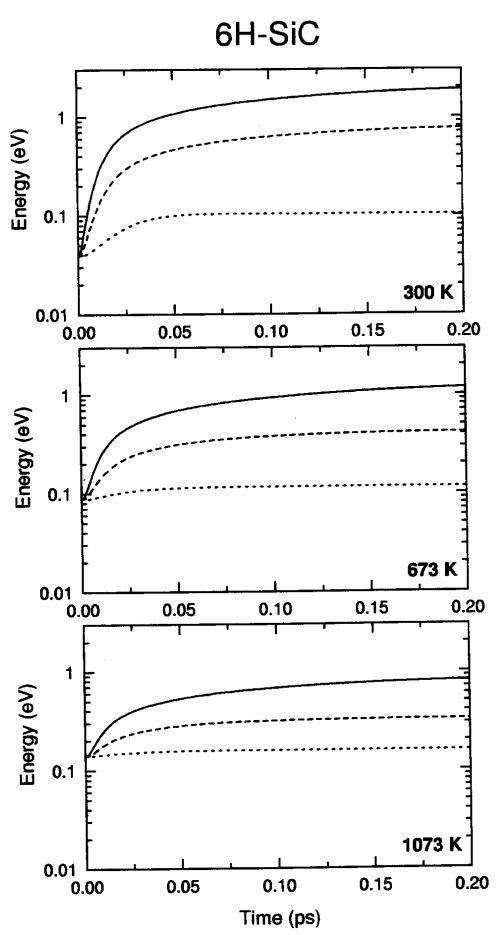

Figure 4. Subpicosecond behavior of the mean electron energy in $6 \mathrm{H}-\mathrm{SiC}$ for lattice temperatures of $300 \mathrm{~K}$ (top), $673 \mathrm{~K}$ (middle), and $1073 \mathrm{~K}$ (bottom). The applied electric field intensities are $200 \mathrm{kV} / \mathrm{cm}$ (dotted line), $600 \mathrm{kV} / \mathrm{cm}$ (dashed line), and $1000 \mathrm{kV} / \mathrm{cm}$ (solid line).

\section{Summary}

The high-field transport behavior in the transient regime of electrons in $3 \mathrm{C}-\mathrm{SiC}$ and $6 \mathrm{H}-\mathrm{SiC}$ at lattice temperatures of $300 \mathrm{~K}, 673 \mathrm{~K}$, and $1073 \mathrm{~K}$ was studied for electric fields intensities up to $1000 \mathrm{kv} / \mathrm{cm}$. The calculations were performed within a simple equivalent isotropic valley picture, and nonparabolic band effects were considered through the hyperbolic model. The 
transient regime was shown to occur in less than $0.3 \mathrm{ps}$ for both $\mathrm{SiC}$ polytypes. It was demonstrated also the possibility of existence of an overshoot in the electron drift velocity, which depends strongly on the applied electric field intensity, the lattice temperature and the $\mathrm{SiC}$ polytype. The overshoot effect presents a remarkable reduction as the lattice temperature is raised. For the electron mean energy, it was shown that its growth rate is substantially reduced with the increase of the lattice temperature.

\section{Acknowledgments}

E. F. Bezerra and E. W. S. Caetano are sponsored by graduate fellowships from the Brazilian National Research Council (CNPq) at the Physics Department of the Universidade Federal do Ceará. V. N. F. and E. F. S. Jr. would like to acknowledge the financial support received during the development of this work from the Science Funding Agencies of the Ceará and Pernambuco states in Brazil (FUNCAP and FACEPE, respectively), the Brazilian National Research Council (CNPq), and the Ministry of Planning (FINEP) under contract PADCT/SDRI \# 77.97.1120.00 3235/97.

\section{References}

[1] W. R. L. Lambrecht and B. Segall, Phys. Rev. B52, R2249 (1995); B. Wenzien, P. Käckell, and F. Bechst- edt, Phys. Rev. B 52, 10897 (1995); C. H. Park, B.-H. Cheong, K.-H. Lee, and K. J. Chang, Phys. Rev. B 49, 4485 (1994); P. Käckell, B. Wenzien, and F. Bechstedt, Phys. Rev. B 50, 10761 (1995); and references therein.

[2] D. K. Ferry, Phys. Rev. B 12, 2361 (1975).

[3] K. Tsukioka, D. Vasileska, and D. K. Ferry, Physica B 185, 466 (1993).

[4] R. P. Joshi, J. Appl. Phys. 78, 5518 (1995).

[5] H.-E. Nilsson, U. Sannemo, and C. S. Patersson, J. Appl. Phys. 80, 3365 (1996).

[6] R. Mickevičius and J. H. Zhao, J. Appl. Phys. 83, 3161 (1998).

[7] W. von Muench and E. Pettenpaul, J. Appl. Phys. 48, 4823 (1977).

[8] X. M. Weng and H. L. Cui, Phys. Stat. Sol. (b) 201, $161(1997)$.

[9] E. O. Kane, J. Phys. Chem. Solids 1, 249 (1957).

[10] M. Shur, Electron. Lett. 12, 615 (1976).

[11] J. P. Nougier, J. C. Vaissiere, D. Gasquet, J. Zimmermann, and E. Constant, J. Appl. Phys. 52, 825 (1981).

[12] B. Carnez, A. Cappy, A. Kaszynski, E. Constant, and G. Salmer, J. Appl. Phys. 51, 784 (1980).

[13] E. W. S. Caetano, E. A. Mendes, V. N. Freire, J. A. P. da Costa, and X. L. Lei, Phys. Rev. B 57, 11872 (1998).

[14] S. Tiwari, Compound Semiconductor Device Physics (Academic Press, San Diego, 1992).

[15] P. Y. Yu and M. Cardona, Fundamentals of Semiconductors (Springer Verlag, Berlin, 1996). 